\title{
THE GENERATION AND DISSIPATION OF INTERSTELLAR TURBULENCE: RESULTS FROM LARGE-SCALE HIGH-RESOLUTION SIMULATIONS
}

\author{
Miguel A. De Avillez ${ }^{1,2}$ AND Dieter BreitschwerdT ${ }^{2}$ \\ Received 2006 December 4; accepted 2007 June 28; published 2007 July 31
}

\begin{abstract}
We study, by means of adaptive mesh refinement hydro- and magnetohydrodynamic simulations that cover a wide range of scales (from kiloparsec to subparsec), the dimension of the most dissipative structures and the injection scale of turbulent interstellar gas, which we find to be about $75 \mathrm{pc}$, in agreement with observations. This is, however, smaller than the average size of superbubbles but consistent with significant density and pressure changes in the ISM, which leads to the breakup of bubbles locally and hence to the injection of turbulence. The scalings of the structure functions are consistent with log-Poisson statistics of supersonic turbulence, where energy is dissipated mainly through shocks. Our simulations are different from previous ones by other authors, since (1) we do not assume an isothermal gas but have temperature variations of several orders of magnitude, and (2) we have no artificial forcing of the fluid with some ad hoc Fourier spectrum but drive turbulence by stellar explosions at the Galactic rate, self-regulated by density and temperature thresholds imposed on the ISM gas.
\end{abstract}

Subject headings: Galaxy: evolution — Galaxy: general — Galaxy: structure — hydrodynamics — ISM: structure

\section{INTRODUCTION}

Long ago it was recognized that interstellar gas is a highly turbulent and compressible medium (von Weizsäcker 1951), although this fact is still being ignored in many theoretical models. Indeed, high-resolution observations of the interstellar medium (ISM) show structures on all scales down to the smallest resolvable ones, implying a dynamical coupling over a wide range of scales, which is a main characteristic of a turbulent flow with Reynolds numbers on the order of Re $=10^{5}-10^{7}$ (cf. Elmegreen \& Scalo 2004). It was not until the last decade that the effects of turbulence in the ISM in general (e.g., Vázquez-Semadeni et al. 1995, 2000; Passot et al. 1995; Korpi et al. 1999; Wada \& Norman 2001; de Avillez \& Mac Low 2002; de Avillez \& Breitschwerdt 2004, 2005, hereafter referred to as AB04 and AB05; Joung \& Mac Low 2006) and in molecular clouds in particular (e.g., Mac Low et al. 1998; Stone et al. 1998; Porter et al. 1998, 1999; Padoan \& Nordlund 1999; Boldyrev et al. 2002; Padoan et al. 2004) have been studied in high detail by means of numerical simulations. The molecular cloud studies focused on decaying and continuously driven turbulence by means of largescale incompressible forcing in Fourier space, stirring the gas in the entire computational domain simultaneously. From these simulations it became clear that (1) isothermal supersonic turbulent motions inside molecular clouds decay within a sound crossing time, becoming subsonic whether a magnetic field is present or not (Mac Low et al. 1998; Stone et al. 1998; Padoan \& Nordlund 1999 ) and (2) the fractal dimension of the most dissipative structures in molecular clouds evolves from close to 1 (filaments) to approximately 2 (shocks) with increasing Mach number (Padoan et al. 2004).

Although there has been a lot of observational, theoretical, and computational research concerning interstellar turbulence, little is known about (1) the scales at which energy is injected and (2) the dimension of the most dissipative structures, if a wide range of scales (from kiloparsec to subparsec) is taken

\footnotetext{
${ }^{1}$ Department of Mathematics, University of Évora, R. Romão Ramalho 59, 7000 Évora, Portugal.

${ }^{2}$ Institut für Astronomie, University of Vienna, Türkenschanzstrasse 17, A-1180 Vienna, Austria; mavillez@astro.univie.ac.at, breitschwerdt@astro.univie.ac.at.
}

into account and the turbulence forcing comprises point explosions acting in small regions of the domain, as opposed to incompressible forcing over the entire domain.

Here we address these issues by means of three-dimensional (3D) adaptive mesh refinement (AMR) hydro- (HD) and magnetohydrodynamic (MHD) simulations of the supernova (SN)driven ISM. The outline of the present Letter is as follows: $\S 2$ deals with the physical model, setup of the simulations, and numerical code used; in $\S 3$ a characterization of the source and injection scale of the interstellar turbulence is made, while in $\S 4$ the Hausdorff dimension of the most dissipative structures is analyzed; $\S 5$ closes the Letter with a discussion and conclusions.

\section{MODEL AND SIMULATIONS}

We use previously published (AB04; AB05) 3D AMR HD and MHD simulations (denoted by HD04 and MHD05) and a new 0.625 pc HD run (denoted by HD06) with self-gravity of the SN-driven ISM in a section of the Galaxy centered at the solar circle having an area of $1 \mathrm{kpc}^{2}$ and extending up to $10 \mathrm{kpc}$ on either side of the Galactic midplane, capturing both the largescale disk-halo-disk circulation and the smallest structures in the Galactic disk. All the runs use a $10 \mathrm{pc}$ resolution coarse grid and generate subgrids up to a local resolution of $1.25 \mathrm{pc}$ (three levels of refinement; HD04 and MHD05 runs) and $0.625 \mathrm{pc}$ (four levels of refinement; HD06 run) in the disk region $|z| \leq$ $500 \mathrm{pc}$ (corresponding to effective grids of $800^{3}$ and $1600^{3}$ cells, respectively), and $2.5 \mathrm{pc}$ (two levels of refinement) in the remaining computational domain. The boundary conditions are periodic along the vertical faces and outflow on the top and bottom faces of the grid.

The physical model, which does not include heat conduction (because we find that turbulent diffusion is a significantly more efficient transport process), includes a gravitational field provided by the stars in the disk (Kuijken \& Gilmore 1989), local self-gravity (excluding the contribution from the newly formed stars), radiative cooling assuming collisional ionization equilibrium (following Dalgarno \& McCray [1972] and Sutherland \& Dopita [1993] cooling functions for gas with $10 \mathrm{~K} \leq T<$ $10^{4} \mathrm{~K}$ and $10^{4} \mathrm{~K} \leq T \leq 10^{8.5} \mathrm{~K}$, respectively) and solar abundances (Anders \& Grevesse 1989), uniform heating due to a 

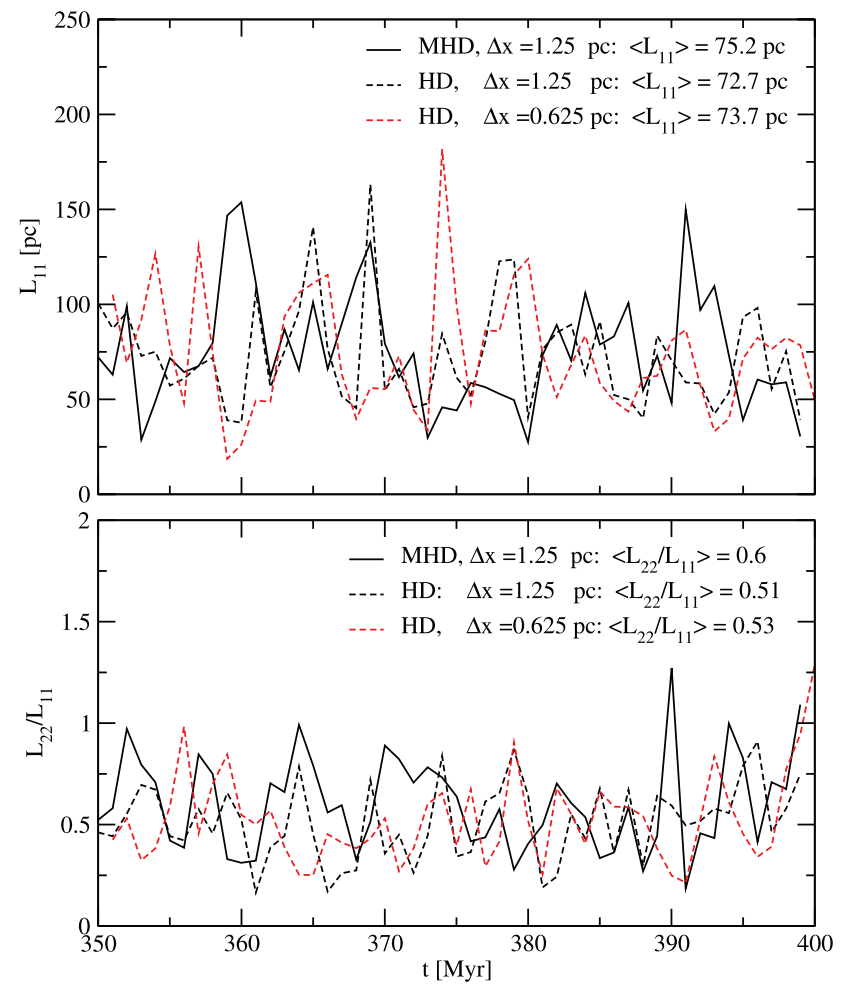

FIG. 1.-History of the characteristic size (given by $L_{11}$ ) of the larger eddies (top) and of the ratio $L_{22} / L_{11}$ (bottom) for the MHD (solid line) and $\mathrm{HD}$ (dashed lines) runs for $1.25 \mathrm{pc}$ (black lines) and $0.625 \mathrm{pc}$ (red lines) resolutions.

UV radiation field normalized to the Galactic value and varying with $z$, and photoelectric heating of grains and polycyclic aromatic hydrocarbons (Wolfire et al. 1995). The disk gas is driven by SN Types Ia and II (including Types Ib, Ic, and II) with the observed Galactic SN rates (Cappellaro et al. 1999). $\mathrm{SNe}$ Ia are set up randomly with an exponential distribution, having a scale height of $325 \mathrm{pc}$ (Freeman 1987). The number and masses of SNe II progenitors are determined according to the local mass distribution in the highest density $\left(n>10 \mathrm{~cm}^{-3}\right)$ cold $(T \leq 100 \mathrm{~K})$ regions of converging flows $(\nabla \cdot v<0$; here $\boldsymbol{v}$ is the gas velocity) on the level 1 grid (note that the coarse grid is identified as level 0) after synchronization of the time steps of the finer and coarser grids. In the flagged regions, with a star-forming efficiency of $10 \%$, the available mass that can be turned into stars is $>840 M_{\odot}$. The masses and number of the new stars are determined by an initial mass function with lower and upper mass cutoffs of 8 and $60 M_{\odot}$, respectively, in order to guarantee that all the available mass goes into highmass $\left(>8 M_{\odot}\right)$ stars. For the present work low-mass stars, which should be formed coevally, are not modeled, but they should have a minor influence (except for the stellar gravitational field, which has already been accounted for) on the ISM dynamics.

The newly formed stars, to which the observed mean random velocity of $5 \mathrm{~km} \mathrm{~s}^{-1}$ is added at the time of formation, are followed kinematically until the end of their main-sequence lifetime determined from Stothers's (1972) formula. The rate at which these stars explode is normalized to the Galactic rate; that is, if the SN rate in the disk is larger/smaller by $10 \%$ of the observed value, SN occurrences are reduced/forced artificially. In general $40 \%-50 \%$ of the Type II SN progenitors explode in the field, while the remaining explosions are cor- related in time and space, generating large superbubble structures, some of which have sizes as large as 500 pc (see AB04; AB05).

The simulations follow a single fluid with an initial vertical density distribution that includes the contributions due to the molecular, neutral, ionized, and hot phases observed in the ISM (Ferrière 2001). The mean magnetic field component in the MHD05 run is initialized assuming equipartition, while the random component, $\delta \boldsymbol{B}$, is 0 . During the first $20 \mathrm{Myr}, \delta \boldsymbol{B}$ builds up, and the total field becomes $4.5 \mu \mathrm{G}$ (AB05).

We use the 3D Évora-Vienna Astrophysics Fluid-Parallel AMR code originally developed by de Avillez (2000). It is a FORTRAN 95 code that solves HD and MHD problems with AMR (Pember et al. 1995; Balsara 2001) in a parallel fashion and uses approximate Riemann solvers for the hydrodynamic and magnetic components (Collela \& Woodward 1984; Dai \& Woodward 1994, 1998).

\section{THE INJECTION SCALE OF INTERSTELLAR TURBULENCE}

The simulations were run for $400 \mathrm{Myr}$, a time sufficiently long to wipe out any signatures of the initial conditions, which are still present at $80 \mathrm{Myr}$ of evolution (see AB04). The initially unstable vertical gas distribution reaches a global dynamical equilibrium at $\sim 200 \mathrm{Myr}$ after the setup of the Galactic fountain, with hot gas ascending into the halo where it cools and finally returns to the disk. During the dynamical equilibrium evolution the Galactic midplane is filled with a thin cold layer overlaid by a thick frothy disk composed mainly of warm neutral and ionized gas having scale heights of 180 and 900 pc, respectively, resembling closely the Lockman (Lockman et al. 1986) and Reynolds (1987) layers. In the disk the highest density (and lowest temperature, if the gas had enough time to cool) gas tends to be confined to sheetlike structures (2D), which are formed by SN-driven, shock-compressed layers. In the HD runs the $T<10^{3} \mathrm{~K}$ gas has no preferred morphology, while in the MHD05 run it tends to be aligned with the magnetic field. The highest densities in the present simulations can be up to $10^{4} \mathrm{~cm}^{-3}$ as a result of the self-gravity occurring in the cold clouds, which are mainly formed in regions where several streams of convergent flows meet. The orientation of these streams is random. We note in passing that if clouds are hit by shocks from random directions, turbulence in the interior is generated. Tapping the turbulent ISM energy reservoir, which is huge, represents a neat way of sustaining supersonic turbulence inside molecular clouds against efficient dissipation.

The scale at which energy is transferred to the interstellar gas can be determined by using the so-called two-point correlation function $R_{i j}(\boldsymbol{l}, t)=\left\langle u_{i}(\boldsymbol{x}+\boldsymbol{l}, t) u_{j}(\boldsymbol{x}, t)\right\rangle$, where $u_{i}$ and $u_{j}$ are the components of the fluctuating velocity field $\boldsymbol{u}$. The diagonal components of $R_{i j}$ are even functions of $\boldsymbol{l}$ and can be written in terms of the dimensionless scalar functions $f(l, t)$ and $g(l, t)$, where $l=\|l\|$, which satisfy $f(0, t)=g(0, t)=$ 1 and $f, g \leq 1$, since $R_{11} / u^{2}=f(l, t)$ and $R_{i j} / u^{2}=g(l, t)$ if $i=j \neq 1$ and 0 if $i \neq j$, with $u=\left(\frac{1}{3}\langle\boldsymbol{u}\rangle\right)^{1 / 2}$. The characteristic injection scale in the flow is given by $L_{11}=\int_{0}^{+\infty} f(l, t) d l$, which in the present simulations is calculated in a region with a linear size of $500 \mathrm{pc}$ at a distance of $250 \mathrm{pc}$ from the edges of the computational domain to avoid the periodicity effects of the boundary conditions. Figure 1 (top) shows the history of $L_{11}$ in the last $50 \mathrm{Myr}$ of evolution of the simulated ISM for 1.25 (black lines) and $0.625 \mathrm{pc}$ (red lines) resolutions. The time average of $L_{11}$ varies between 73 and $75 \mathrm{pc}$ for the HD and 


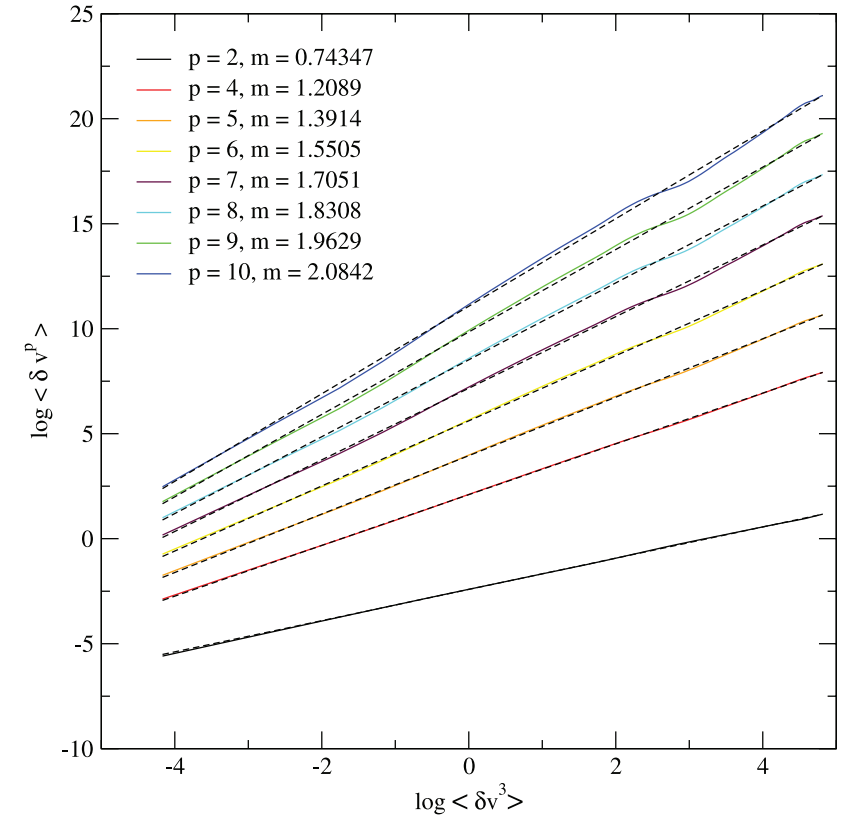

FIG. 2.- Log-log plot of the velocity correlation moments $\left\langle\delta v_{l}^{p}\right\rangle$ as a function of $\left\langle\delta v_{l}^{3}\right\rangle$ and best fits (dashed lines) for $p=2,4, \ldots, 10$. The data refer to the $0.625 \mathrm{pc}$ resolution run.

MHD cases, respectively, and seems to be independent of resolution. In all the cases considered here, there is a large scatter of $L_{11}$ around its mean as a result of the expansion of bubbles and superbubbles in an inhomogeneous medium characterized by a large number of SNe exploding in the field and the merging of bubbles and superbubbles. This scale corresponds to the one at which the power spectrum of the solenoidal component of the velocity field has a spectral break, which we interpret as a redistribution of energy to both larger and smaller scales (M. A. de Avillez \& D. Breitschwerdt 2007, in preparation).

The ratio between the transverse, $L_{22}=\int_{0}^{+\infty} g(l, t) d l$, and longitudinal correlation lengths $\left(L_{22} / L_{11}\right.$, which in isotropic turbulence is 0.5 ) varies between 0.2 and 1.3 (Fig. 1, bottom), with its time average over a period of $50 \mathrm{Myr}$ being $\left\langle L_{22} / L_{11}\right\rangle_{t} \sim 0.5$ (HD04 and HD06 runs) and 0.6 (MHD05 run). The former value indicates that in a statistical sense the interstellar unmagnetized turbulence is roughly isotropic, while the $20 \%$ deviation from 0.5 seen in the MHD05 run suggests that $\left\langle L_{22} / L_{11}\right\rangle_{t}$ increases with magnetic field strength. This is also supported by similar increases observed in other MHD runs that we carried out $(0.25,0.5,1,1.5$, and $2 \mu \mathrm{G})$, which will be described in a forthcoming paper.

\section{INTERMITTENCY AND HAUSDORFF DIMENSIONS}

From the Kolmogorov (1941, hereafter K41) model for homogeneous and isotropic turbulence, one can derive that, in the inertial range, the moments of order $p,\left\langle\delta v_{l}^{p}\right\rangle$, are dictated solely by $\left\langle\delta v_{l}^{3}\right\rangle$ via the set of scalings $\zeta(p) / \zeta(3)$ :

$$
\left\langle\delta v_{l}^{p}\right\rangle \propto\left\langle\delta v_{l}^{3}\right\rangle^{\zeta(p) / \zeta(3)},
$$

with $p>1$ being an integer. In this expression $\delta v_{l}=v(x+$ $l)-v(x)$, with $v(x+l)$ and $v(x)$ being the velocities along the $x$-axis at two points separated by a distance $\eta \ll l \ll L_{11}$ and with $L_{11}$ being the outer scale and $\eta=\nu^{3 / 4} \epsilon^{-1 / 4}$ the Kolmogorov
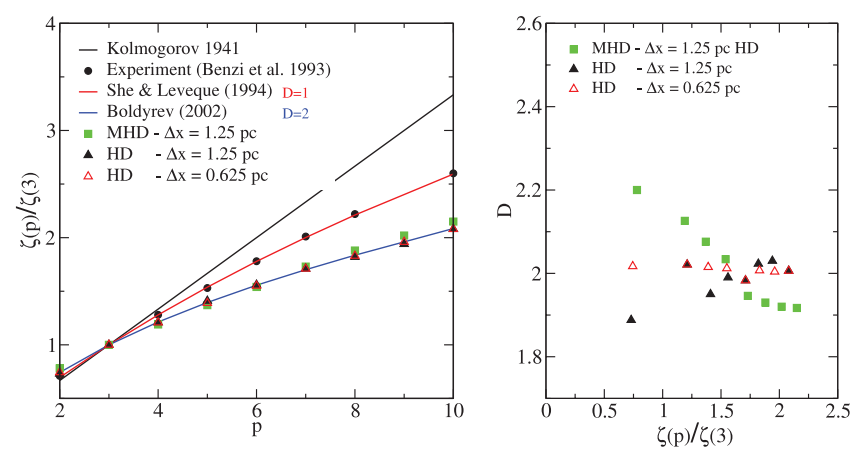

Fig. 3.-Left: Exponent $\zeta(p) / \zeta(3)$ for the structure function vs. order $p$. The solid line corresponds to $\zeta(p)=p / 3$ (K41 theory), circles correspond to the data of Benzi et al. (1993), and red and blue lines refer to the She-Levéque (SL94) and Burgers-Kolmogorov (BK02) models, respectively. Triangles represent data from HD simulations with $1.25 \mathrm{pc}$ (black triangles) and $0.625 \mathrm{pc}$ (red triangles) resolutions, and the green squares refer to the MHD run with $1.25 \mathrm{pc}$ resolution. Right: Fractal dimension $D$ of the most dissipative structures (derived from eq. [2]) as a function of the exponent $\zeta(p) / \zeta(3)$, with $p=2,4$, $\ldots, 10$, for the three simulations.

microscale; $\langle\ldots\rangle$ stands for the ensemble average over the probability density function of $\delta v_{l}$. Experiments have shown that (see discussions in Benzi et al. 1993 and Frisch 1995) (1) equation (1), with the same scaling exponents, is valid in a wide range of length scales for large as well as small Re, even if no inertial range is established, and (2) in the inertial range of high-Re flows the scaling exponents become significantly smaller than the linear law $p / 3$ (the K41 scaling) with increasing $p$. This is interpreted as the statistics of small scales (the ones dictating the behavior of higher order moments) becoming increasingly non-Gaussian.

In the unmagnetized and magnetized simulated interstellar turbulence driven by point explosions the moments $\left\langle\delta v_{l}^{p}\right\rangle$ are related to $\left\langle\delta v_{l}^{3}\right\rangle$ [see Fig. 2, which shows the moments and the slopes $\zeta(p) / \zeta(3)$ of their best fits for the HD06 run], supporting the validity of equation (1) in such cases of compressible forcing.

Figure 3 (left) compares theoretical, experimental, and simulated results (black and red triangles refer to the HD04 and HD06 runs, respectively, while squares refer to the MHD05 run) on the variation of $\zeta(p) / \zeta(3)$ with $p$. The experimental data are taken from Benzi et al. (1993), and the theoretical predictions correspond to the K41 and She \& Levéque (1994, hereafter SL94) models of incompressible turbulence and the Burgers-Kolmogorov model (Boldyrev 2002, hereafter BK02) for supersonic turbulence. The K41 model considers lognormal statistics for the transfer of energy from large to small scales and has no corrections for intermittency. The SL94 model uses log-Poisson statistics to describe intermittency and assumes that energy dissipation occurs through filamentary structures (Hausdorff dimension $D=1$ ) resulting from vortex stretching. The BK02 and SL94 models differ in the geometry of the most intermittent structures, which for supersonic turbulence are assumed to be shocks (Hausdorff dimension $D=2$ ). We note that the geometry of those scales does not have to represent dissipative structures (as in incompressible turbulence) nor do they have to be planar. Two dimensions in the Hausdorff sense could represent various structures, sheets being the simplest. This is further supported by the fact that Burger's turbulence produces discontinuities but not shocks, in the sense that material cannot flow through them; they just sweep up material. 
The scalings $\zeta(p) / \zeta(3)$ can be written as (Dubrulle 1994; see the discussion in Politano \& Pouquet 1995)

$$
\frac{\zeta(p)}{\zeta(3)}=\frac{p}{g}(1-x)+(3-D)\left(1-\beta^{p / g}\right),
$$

where $g$ is linked to the model of nonlinear energy transfer via the basic scaling $v_{l} \sim l^{1 / g}$ and $x$ is related to the energy transfer time at the smallest scale, $t_{l} \sim l^{x}$, with $x>0$ and $\beta=1-$ $x /(3-D)$. In these expressions $D$ is the fractal dimension of the most intermittent structures. In the present simulations turbulence is mostly solenoidal in the inertial range, and therefore, $g=3$ and $x=\frac{2}{3}$. Thus, the Hausdorff dimension $D$ of the most intermittent structures derived from equation (2) and using the $\zeta(p) / \zeta(3)$ values shown in Figure 3 (left) varies between 1.9 and $2.2(D=1.98-2.02,1.9-2.03$, and $1.91-2.2$ in the HD06, HD04, and MHD05 runs, respectively; Fig. 3, right). These values suggest that the energy injected by $\mathrm{SNe}$ into interstellar turbulence is dissipated preferentially through $2 \mathrm{D}$ structures that can be identified as shock surfaces in the HD cases and also as current sheets in the MHD case (see Müller \& Biskamp 2000). The discrepancy observed in the MHD run, although within the errors of numerical noise, indicates a tendency toward filamentary dissipative structures due to the anisotropy induced by the magnetic field. We postpone a more detailed discussion on this issue to a forthcoming paper.

\section{DISCUSSION AND CONCLUSIONS}

Contrary to the previous work of other authors, the present runs (1) incorporate the full-extent disk-halo-disk circulation, (2) do not assume isothermality (temperature varies within several orders of magnitude), (3) include magnetic fields and selfgravity, and (4) cover a wide range of scales (from kiloparsec to subparsec), providing resolution-independent information on the injection scale, extended self-similarity, and dissipative structures. The mean integral scale at which energy is injected into the interstellar medium is on the order of $75 \mathrm{pc}$. This scale is significantly lower than the often quoted average size of evolved SNRs or superbubbles, because energy is already injected at the timescale of breakup of the remnants due to density and pressure gradients in the inhomogeneous ambient medium, which occurs well before they stall. The similarity between the HD and MHD results indicates that magnetic pressure and tension forces cannot prevent breakout of the hot gas from the bubbles formed by SN explosions, as long as $L \lesssim \beta_{P} \xi$, where $L$ and $\xi$ are the scale lengths of thermal and magnetic pressures (including tension forces) and $\beta_{P}=4 \pi P / B^{2}$ is the plasma beta. However, the magnetic field introduces a degree of anisotropy which is reflected in the $20 \%$ deviation from 0.5 seen in the time-averaged ratio $L_{22} / L_{11}$ of the magnetized ISM. Kaplan (1958) already suggested an injection scale of $80 \mathrm{pc}$ based on the analysis of second-order structure functions of interstellar matter. The dissipation of energy in our interstellar turbulence simulations proceeds through shocks and numerical viscosity, as we cannot resolve the physical viscosity scales. The variation of $\zeta(p) / \zeta(3)$ with $p$ is most consistent with a log-Poisson model for the scales at which intermittency becomes important.

Recently, Joung \& Mac Low (2006) found similar scalings in a SN-driven ISM HD simulation, although they did not consider self-gravity, and found a hot $\left(T>10^{5.5} \mathrm{~K}\right)$ gas occupation fraction $f_{h}>40 \%$, which is at odds with observations and other models. Their resolution $(1.95 \mathrm{pc})$ in the disk is a factor of 3 lower than that in the HD06 run $(0.625 \mathrm{pc})$. Their high value of $f_{h}$ implies that the amount of $\mathrm{O}$ VI in the ISM is at least a factor of 2 larger than that observed with Copernicus and FUSE. According to our simulations, reliable comparisons of global properties with observations (e.g., occupation fractions) can only be made after the system reaches a dynamical equilibrium $(t>150 \mathrm{Myr})$, implying a relaxation time much larger than a few million years (see discussion in AB04).

We thank the referee and the editor for detailed reports that allowed us to significantly improve the Letter. This research is supported by the Portuguese Science Foundation through project POCTI/FIS/58352/2004.

\section{REFERENCES}

Anders, E., \& Grevesse, N. 1989, Geochim. Cosmochim. Acta, 53, 197 de Avillez, M. A. 2000, MNRAS, 315, 479

de Avillez, M. A., \& Breitschwerdt, D. 2004, A\&A, 425, 899 (AB04) 2005, A\&A, 436, 585 (AB05)

de Avillez, M. A., \& Mac Low, M.-M. 2002, ApJ, 581, 1047

Balsara, D. S. 2001, J. Comput. Phys., 174, 614

Benzi, R., Ciliberto, S., Tripiccione, R., Baudet, C., Massaioli, F., \& Succi,

S. 1993, Phys. Rev. E, 48, 29

Boldyrev, S. 2002, ApJ, 569, 841 (BK02)

Boldyrev, S., Nordlund, Å., \& Padoan, P. 2002, Phys. Rev. Lett., 89, 31102

Cappellaro, E., Evans, R., \& Turato, M. 1999, A\&A, 351, 459

Collela, P., \& Woodward, P. R. 1984, J. Comput. Phys., 54, 174

Dai, W., \& Woodward, P. R. 1994, J. Comput. Phys., 115, 485 1998, J. Comput. Phys., 142, 331

Dalgarno, A., \& McCray, R. A. 1972, ARA\&A, 10, 375

Dubrulle, B. 1994, Phys. Rev. Lett., 73, 959

Elmegreen, B. G., \& Scalo, J. 2004, ARA\&A, 42, 211

Ferrière, K. 2001, Rev. Mod. Phys., 73, 1031

Freeman, K. C. 1987, ARA\&A, 25, 603

Frisch, U. 1995, Turbulence (Cambridge: Cambridge Univ. Press)

Joung, M. K. R., \& Mac Low, M.-M. 2006, ApJ, 653, 1266

Kaplan, S. A. 1958, in IAU Symp. 6, Electromagnetic Phenomena in Cosmical Physics, ed. B. Lehnert (Cambridge: Cambridge Univ. Press), 504

Kolmogorov, A. N. 1941, CR Acad. Sci. URSS, 30, 301 (K41)

Korpi, M. J., Brandenburg, A., Shukurov, A., Tuominen, I., \& Nordlund, Å. 1999, ApJ, 514, L99
Kuijken, K., \& Gilmore, G. 1989, MNRAS, 239, 651

Lockman, F. J., Hobbs, L. M., \& Shull, J. M. 1986, ApJ, 301, 380

Mac Low, M.-M., Klessen, R. S., Burkert, A., \& Smith, M. D. 1998, Phys. Rev. Lett., 80, 2754

Müller, W.-C., \& Biskamp, D. 2000, Phys. Rev. Lett., 84, 475

Padoan, P., Jimenez, R., Nordlund, Å., \& Boldyrev, S. 2004, Phys. Rev. Lett., 92, 1102

Padoan, P., \& Nordlund, Å. 1999, ApJ, 526, 279

Passot, T., Vázquez-Semadeni, E., \& Pouquet, A. 1995, ApJ, 455, 536

Pember, R. B., Bell, J. B., Colella, P., Crutchfield, W. Y., \& Welcome, M. L. 1995, J. Comput. Phys., 120, 278

Politano, H., \& Pouquet, A. 1995, Phys. Rev. E, 52, 636

Porter, D. H., Pouquet, A., Sytine, I., \& Woodward, P. R. 1999, Physica A, 263, 263

Porter, D. H., Woodward, P. R., \& Pouquet, A. 1998, Phys. Fluids, 10, 237

Reynolds, R. J. 1987, ApJ, 323, 118

She, Z.-S., \& Levèque, E. 1994, Phys. Rev. Lett., 72, 336 (SL94)

Stone, J. M., Ostriker, E. C., \& Gammie, C. F. 1998, ApJ, 508, L99

Stothers, R. 1972, ApJ, 175, 431

Sutherland, R. S., \& Dopita, M. A. 1993, ApJS, 88, 253

Vázquez-Semadeni, E., Gazol, A., \& Scalo, J. 2000, ApJ, 540, 271

Vázquez-Semadeni, E., Passot, T., \& Pouquet, A. 1995, ApJ, 441, 702

von Weizsäcker, C. F. 1951, ApJ, 114, 165

Wada, K., \& Norman, C. A. 2001, ApJ, 547, 172

Wolfire, M. G., McKee, C. F., Hollenbach, D., \& Tielens, A. G. G. M. 1995, ApJ, 453, 673 